\title{
Association of Underlying Diseases and Clinical Characteristics with Mortality in Patients with 2019 Novel Coronavirus in Iran
}

\author{
Mohammadhossein Zamanian ${ }^{1}$, Zohre Foroozanfar ${ }^{2,{ }^{*}}$, Zhila Izadi $^{3,{ }^{* *}}$, Samira Jafari ${ }^{3}$, Hossein \\ Derakhshankhah ${ }^{3}$, Mehdi Salimi ${ }^{1}$, Alireza Janbakhsh (iD ${ }^{1}$, Babak Sayad (iD) ${ }^{1}$, Maria Shirvani (iD) ${ }^{1}$, Seyed \\ Vahid Jasemi ${ }^{1}$, Feyzollah Mansouri (iD ${ }^{1}$, Mandana Afsharian ${ }^{(10}{ }^{1}$, Zeinab Mohseniafshar $^{1}$, Ronak Miladi $^{1}$ \\ and Siavash Vaziri (iD) 1
}

${ }^{1}$ Infectious Diseases Research Center, Kermanshah University of Medical Sciences, Kermanshah, Iran

${ }^{2}$ Department of Epidemiology and Biostatistics, School of Public Health, Tehran University of Medical Sciences, Tehran, Iran

${ }^{3}$ Pharmaceutical Sciences Research Center, Health Institute, Kermanshah University of Medical Sciences, Kermanshah, Iran

"Corresponding author: Department of Epidemiology and Biostatistics, School of Public Health, Tehran University of Medical Sciences, Tehran, Iran. Email: foroozanfar.zohre@gmail.com

${ }^{* *}$ Corresponding author: : Pharmaceutical Sciences Research Center, Health Institute, Kermanshah University of Medical Sciences, Kermanshah, Iran. Email izadi_zh@razi.tums.ac.ir

Received 2020 May 06; Revised 2020 May 23; Accepted 2020 June 07.

\begin{abstract}
Objectives: The first case of 2019 novel coronavirus disease (COVID-19) was reported in Iran in February 2020. Here, we report the epidemiological and clinical characteristics of patients with COVID-19 and factors associated with mortality in these patients.

Methods: A retrospective cohort study was conducted from February 22, 2020, to March 24, 2020, in Golestan Hospital in Kermanshah, Iran. Demographic data including underlying diseases and clinical data including the presenting symptoms, chest computed tomography (CT) scan, reverse transcription polymerase chain reaction (RT-PCR) test results, and outcomes were extracted from electronic medical records. Simple and multiple logistic regression methods were used to explore the factors associated with mortality.

Results: Of 245 patients admitted with COVID-19, 155 (63.30\%) were male. The mean age of the subjects was $54.68 \pm 19.21$. Forty-five (18.48\%) patients had underlying diseases. Common symptoms were dyspnea $(n=137 ; 55.9 \%)$, cough $(n=93 ; 38.0 \%)$, and fever $(n=$ $78 ; 31.8 \%$ ). All patients had pneumonia with abnormal findings on chest CT scan (100\%), and RT-PCR test results were positive in 87 (35.50\%) patients. Of the total admitted cases, 38 (15.5\%) patients died during hospitalization. An old age (OR $=1.09 ; 95 \% \mathrm{CI}: 1.02$ to 1.06), history of heart disease ( $\mathrm{OR}=5.07$; $95 \% \mathrm{CI}: 1.46$ to 17.58$)$, hypertension ( $\mathrm{OR}=5.82 ; 95 \% \mathrm{CI}: 1.13$ to 30.04$)$, smoking $(\mathrm{OR}=11.44 ; 95 \%$ $\mathrm{CI}: 1.01$ to 29.53), history of at least one underlying disease (OR=3.31; 95\%CI: 1.54 to 7.09), and symptoms of decreased consciousness at the time of admission ( $\mathrm{OR}=24.23$; $95 \% \mathrm{CI}: 2.62$ to 223.39) were associated with mortality. Also, the symptoms of $\operatorname{cough}(\mathrm{OR}=0.383$; $95 \% \mathrm{CI}: 0.17$ to 0.88 ) and fever ( $\mathrm{OR}=0.278 ; 95 \% \mathrm{CI}: 0.10$ to 0.74 ) had a negative association with mortality.

Conclusions: In the current study, factors including old age, smoking, symptoms of decreased consciousness, and underlying diseases such as heart disease, hypertension, and history of at least one underlying disease were associated with mortality. Factors associated with mortality should be considered so that we can better manage patients with COVID-19.
\end{abstract}

Keywords: COVID-19, Underlying Diseases, Mortality, Predictors

\section{Background}

The biggest universal event of 2020 has been the coronavirus disease-19 (COVID-19) epidemic. It was reported for the first time in Wuhan, Hubei Province, China, on December 7, according to the official statement of the Chinese Government. As known, COVID-19 belongs to the coronavirus family, which causes infectious diseases such as common cold-like illnesses or, more critical ones, SARS, and MERS. Its common symptoms include fever, dry cough, and shortness of breath. Muscular pain, sputum production, and sore throat are the less common symptoms of COVID-19 infection. Since emergence, it rapidly spread worldwide to become a pandemic disease of undefined origin. Its economic and social impacts may persist even with the disease control (1-3).

According to the latest published reports by the World Health Organization (WHO), by April 27, 2020, COVID-19 caused a large outbreak in at least 213 countries (4). In Iran, the first case was reported in February 2020 in Qom. 
Iran has been assigned to have the most abundant number of patients and deaths (89,328 confirmed cases and 5,650 deaths) due to COVID-19 in this period (4). Although the number of new cases has been increasing day by day, it has been much lower than in some other countries. Overall, a recovery rate of $83.5 \%$ has been reported. In addition, previous studies have shown an association between underlying diseases such as cardiovascular diseases, hypertension, diabetes, and cancer and progression, prognosis, and mortality in COVID-19 patients (5-8).

The diagnosis of COVID-19 can be done by different methods, including the molecular detection of the virus genome by real-time transcription polymerase chain reaction (RT-PCR), clinical symptoms of the patient, chest computed tomography (CT) scan, cell culture, and rapid tests. Laboratory evidence to aid in the diagnosis of this infection includes leukopenia, lymphocytopenia, the increment of C-reactive protein (CRP), lactate dehydrogenase (LDH), and hypoxia (9-11).

According to available guidelines, the gold standard test for the diagnosis of COVID-19 is RT-PCR, but there are limitations in access to diagnostic kits, the low sensitivity of kits, and defects in proper oropharynx sampling in our country, Iran. Therefore, due to the highly contagious nature of the virus and the vital need for identifying and early treatment of patients, chest CT scan as a routine imaging tool to diagnose pneumonia, accompanied by clinical symptoms, is used for the rapid and easy detection of lung involvement, even in patients with negative RT-PCR test results $(12,13)$.

\section{Objectives}

This study aimed to describe the epidemiological and clinical characteristics of COVID-19 patients and the association of underlying diseases and clinical characteristics with mortality.

\section{Methods}

\subsection{Patients}

To organize this retrospective study from a single institution, data were gathered from February 22, 2020, to March 24, 2020, at Golestan Hospital in Kermanshah, Iran. The Golestan Hospital, with two general and Intensive Care Unit (ICU) wards, was officially founded for burn patients on November 20, 2019. However, since the outbreak of COVID-19 in Kermanshah on February 22, 2020, it was provided for the care of COVID-19 patients. The average number of staff in this institution is approximately 25 nurses,
10 general practitioners, one pulmonologist, four infectious disease specialists, and four anesthesiologists. Overall, 3,085 patients presented to the Golestan Hospital Emergency Department between February 22, 2020, and March 24, 2020.

\subsection{Method}

In the current single-center study, the diagnosis and treatment of COVID-19 at outpatient and inpatient levels were conducted based on the designed flowchart in Figure 1. Clinical symptoms (e.g., fever, chills, sore throat, and dry cough), chest CT scan, patterns of pulmonary involvement, oxygen saturation at admission, and RT-PCR assay tests were analyzed for suspected patients, which are explained in detail in the following.

The early diagnosis of COVID-19 was feasible based on the assessment of prevalent clinical symptoms related to this infectious disease reported in the majority of infected people, including fever, chills, sore throat, and dry cough. Therefore, at the first step, these symptoms were examined in all patients. Then, according to imaging findings and admission protocols, a subgroup of these patients was admitted. Of 3,085 enrolled patients, 252 patients were candidates for admission.

\subsection{Data Collection}

In the present study, the demographic and clinical characteristics, history of underlying diseases, presenting symptoms, chest CT scan results, RT-PCR test results, and outcomes (discharge, mortality, and length of stay) were available to be analyzed. Chest CT scan findings indicative of pulmonary involvement and $\mathrm{O}_{2}$ saturations $<90 \%$ were regarded as the eligibility criteria for hospitalization based on the presented flowchart.

\subsection{CT Protocol}

Chest CT scans were performed using two multidetector CT scanners. The chest CT scan is a non-invasive and conventional diagnostic imaging method. The patients were imaged in the supine position using CT scanners (CT Scanner, Toshiba Alexion 16 Slice). To minimize motion artifacts, the patients were instructed to hold their breath. It should be considered to adjust major scanning parameters, as follows: Tube voltage (120 kVp), automatic tube current modulation (30 - $70 \mathrm{mAs}$ ), pitch (0.99 - 1.22 $\mathrm{mm})$, matrix $(512 \times 512)$, slice thickness $(10 \mathrm{~mm})$, and field of view $(350 \mathrm{~mm} \times 350 \mathrm{~mm})$. According to a literature review on viral pneumonia, the recorded chest CT scans were assessed for the characteristics as summarized in Table1. The typical radiographic features were demonstrated 


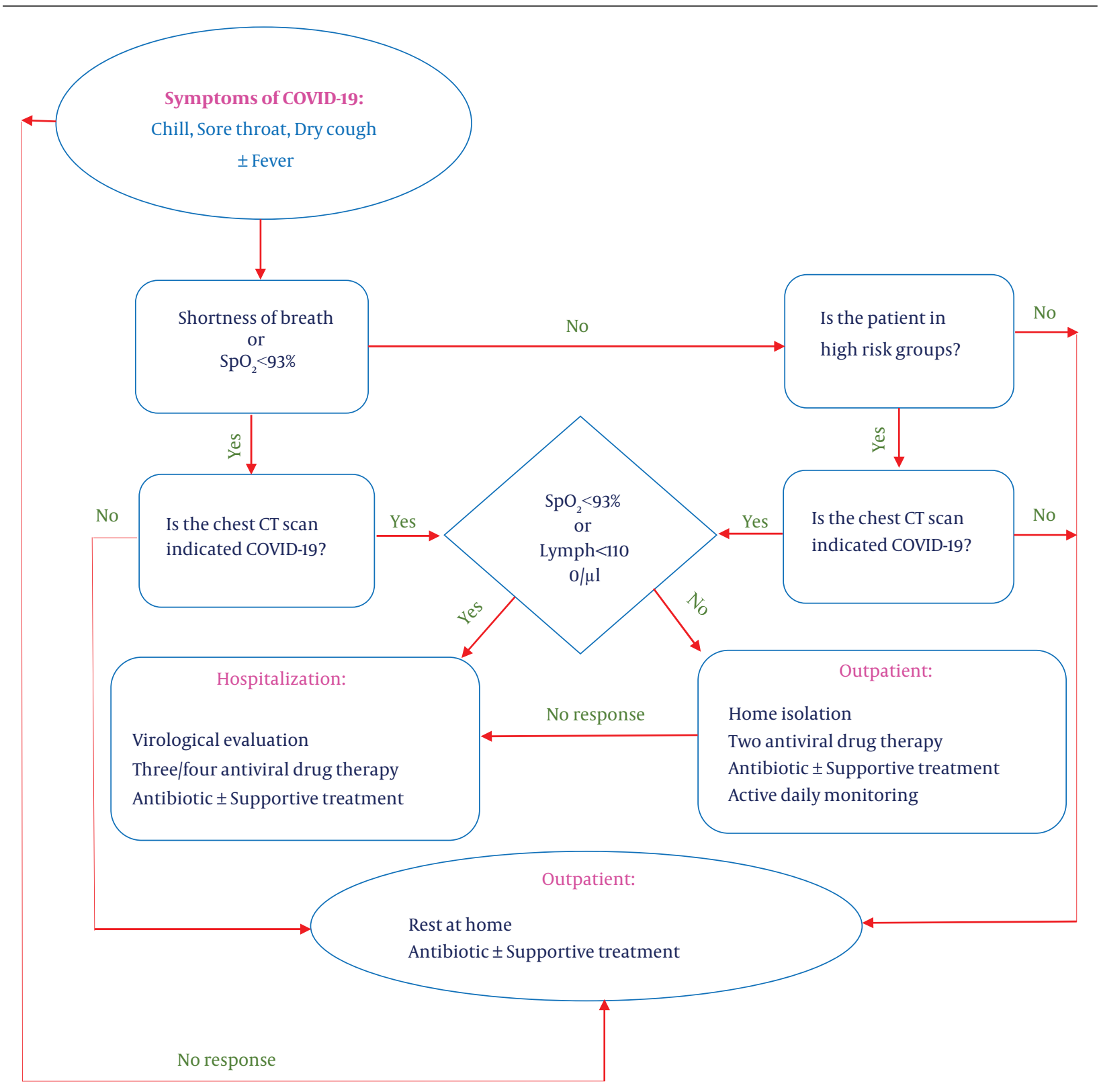

Figure 1. Flowchart for diagnosis and treatment of COVID-19 at outpatient and inpatient levels. Department of Infectious Diseases, Faculty of Medicine, Kermanshah University of Medical Sciences (edition: March 11, 2020).

in almost all of our COVID-19 admitted patients, which included ground-glass opacities in bilateral, sub-pleural, and peripheral regions, interlobular septal thickening, crazypaving patterns, air-space consolidation, and interstitial changes with a peripheral distribution $(14,15)$. Accordingly, all the recorded CT images were scrutinized by an expert radiologist for the interpretation of the obtained data. The final decision on positive/negative CT scan outcomes was announced by consensus. In this study, the chest CT results were compatible with COVID-19 for all patients.

\subsection{Detection of COVID-19 in Plasma (RT-PCR)}

After confirmation of clinical and pulmonary presentations, An RT-PCR assay was conducted on nasal and pharyngeal swab specimens of the suspected patients by a commercial test kit (LightMix ${ }^{\circledR}$ SarbecoV E-gene plus EAVcontrol, TIB MOLBIOL Syntheselabor GmbH, Germany). 


\begin{tabular}{|c|c|c|c|c|}
\hline Characteristics & Patients $(\mathrm{N}=\mathbf{2 4 5})$ & $\operatorname{Dead}(\mathrm{N}=38)$ & Alive $(\mathbf{N}=\mathbf{2 0 7})$ & PValue \\
\hline Age & $54.68 \pm 19.21$ & $65.52 \pm 16.55$ & $52.67 \pm 18.75$ & $0.001^{\mathrm{a}}$ \\
\hline \multicolumn{5}{|l|}{ Age group } \\
\hline $39 \geq$ & $63(25.7)$ & $3(7.9)$ & $60(29.0)$ & $0.006^{\mathrm{a}}$ \\
\hline $40-49$ & $34(13.9)$ & $5(13.2)$ & $29(14.0)$ & \\
\hline $50-59$ & $46(18.8)$ & $6(15.8)$ & $40(19.3)$ & \\
\hline $60-69$ & $49(20.0)$ & $8(21.1)$ & $41(19.8)$ & \\
\hline $70 \leq$ & $53(21.6)$ & $16(42.1)$ & $37(17.9)$ & \\
\hline \multicolumn{5}{|l|}{ Sex } \\
\hline Male & $155(63.3)$ & $27(71.1)$ & $128(61.8)$ & 0.185 \\
\hline Female & $90(36.7)$ & $11(28.9)$ & $79(38.2)$ & \\
\hline \multicolumn{5}{|l|}{ History of underlying disease } \\
\hline Current smoking & $3(1.2)$ & $2(5.3)$ & $1(0.5)$ & 0.064 \\
\hline Diabetes & $9(3.67)$ & $2(5.3)$ & $7(3.4)$ & 0.420 \\
\hline Hypertension & $6(2.44)$ & $3(7.9)$ & $3(1.4)$ & $0.049^{\mathrm{a}}$ \\
\hline Cardiovascular disease & $11(4.48)$ & $5(13.2)$ & $6(2.9)$ & $0.016^{\mathrm{a}}$ \\
\hline Others & $28(11.40)$ & $6(15.8)$ & $22(10.6)$ & 0.251 \\
\hline \multicolumn{5}{|l|}{ Signs and symptoms at admission } \\
\hline Fever & $78(31.8)$ & $5(13.2)$ & $73(35.3)$ & $0.004^{\mathrm{a}}$ \\
\hline Cough & $93(38.0)$ & $8(21.1)$ & $85(41.1)$ & $0.014^{\mathrm{a}}$ \\
\hline Dyspnea & $137(55.9)$ & $25(65.8)$ & $112(54.1)$ & 0.123 \\
\hline Headache & $5(2.0)$ & - & $5(2.4)$ & - \\
\hline Chest pain & $1(0.4)$ & - & $1(0.5)$ & - \\
\hline Diarrhea & $5(2.0)$ & - & $5(2.4)$ & - \\
\hline Vomiting & $4(1.6)$ & $1(2.6)$ & $3(1.4)$ & 0.493 \\
\hline Abdominal pain & $4(1.6)$ & - & $4(1.9)$ & - \\
\hline Fatigue/weakness & $15(6.1)$ & $1(2.6)$ & $14(6.8)$ & 0.291 \\
\hline Nausea & $6(2.4)$ & $1(2.6)$ & $5(2.4)$ & 0.640 \\
\hline Myalgia & $8(3.3)$ & $1(2.6)$ & $7(3.4)$ & 0.640 \\
\hline Anorexia & $5(2.0)$ & $2(5.3)$ & $3(1.4)$ & 0.173 \\
\hline Loss of consciousness & $5(2.0)$ & $4(10.5)$ & $1(0.5)$ & $0.002^{\mathrm{a}}$ \\
\hline Respiratory distress & $12(4.9)$ & $3(7.9)$ & $9(4.3)$ & 0.280 \\
\hline More than one sign or symptom & $128(52.2)$ & $14(36.8)$ & $114(55.1)$ & $0.029^{\mathrm{a}}$ \\
\hline Positive PCR & $87(35.5)$ & $16(42.1)$ & $71(34.3)$ & 0.228 \\
\hline Hospital stay & $3(2.0-5.0)$ & $2(1.0-4.0)$ & $3(2.0-5.0)$ & 0.060 \\
\hline Admitted to ICU & $49(20.0)$ & $15(39.5)$ & $34(16.4)$ & $0.002^{\mathrm{a}}$ \\
\hline
\end{tabular}

${ }^{\mathrm{a}}$ Significant at the 0.05 level.

\subsection{Statistical Analysis}

Qualitative variables were described in terms of numbers and percentages, and quantitative variables were reported as mean and standard deviation or median (IQR).
The Mann Whitney U-test, chi-square test, Fisher's exact test, and $t$-test were used. In addition, simple logistic regression analysis was used to determine the factors associated with mortality in patients with COVID-19, and vari- 
ables with P values of $\leq 0.2$ in the simple analysis were entered into the multiple logistic regression model with a significance level of $\mathrm{P}<0.05$. All statistical analyses were performed using SPSS version 23.0 software.

The funding source refers to the result section: Based on the obtained results from this assay, of 252 patients screened, 158 had negative RT-PCR test results, 87 had positive RT-PCR test results, and seven demonstrated unknown results that were excluded from this study.

\section{Results}

In the present study, 245 patients with COVID-19 were enrolled of whom, 155 (63.30\%) were male, and 90 (36.70\%) were female. The mean age of the subjects was $54.68 \pm$ 19.21, which was $53.54 \pm 17.37$ in males and $56.61 \pm 20.91$ in females; however, the difference was not statistically significant $(P=0.223)$. The number of patients stratified by age groups is shown in Figure 2. According to the results, most patients were in the age group of $\leq 39$ years.

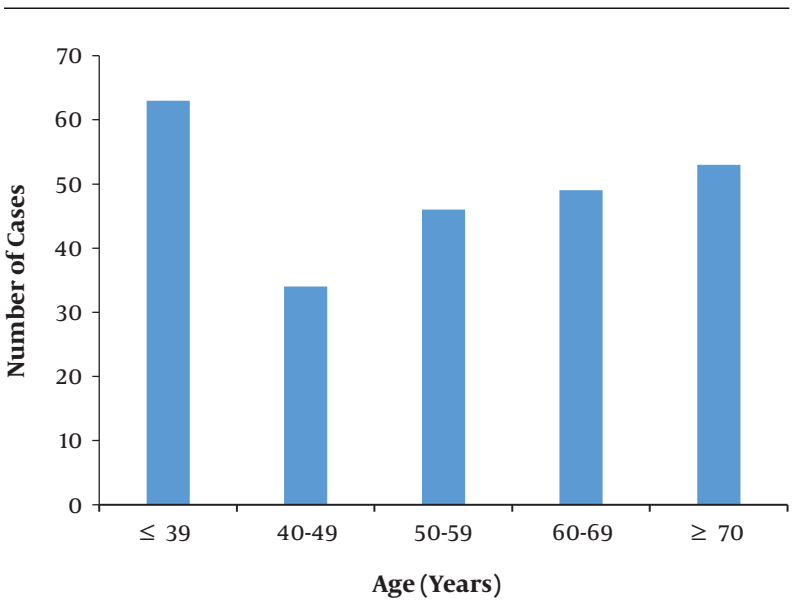

Figure 2. Number of patients with COVID-19 by age groups

The demographic and clinical data of patients stratified by survival are reported in Table 1 . The most common symptoms at the onset of the disease were dyspnea in 137 cases (55.9\%), cough in 93 cases (38.0\%), and fever in 78 cases (31.8\%). The mean hospital stay in discharged patients was $3.45 \pm 1.68$ days, which was $3.52 \pm 1.73$ days in men and 3.32 \pm 1.58 days in women $(\mathrm{P}=0.47)$. Moreover, $49(20 \%)$ patients were admitted to the Intensive Care Unit(ICU), while 196 (80\%) did not receive ICU care. Significant differences were observed in mortality between patients who were admitted to the ICU (30.6\%) and those who were not (11.7\%) $(\mathrm{P}=0.002)$. Forty-five $(18.45 \%)$ patients had a history of at least one underlying disease, including 11 cases with cardiovascular disease (4.48\%), nine (3.67\%) with diabetes, and six (2.44\%) with hypertension. Besides, 38 (15.5\%) patients passed away at the hospital, with the highest mortality rate in the age group of 70 years or older.

The results of simple regression analyses showed that factors such as age, smoking, presenting symptoms of decreased consciousness at admission, history of at least one underlying disease, history of cardiovascular disease, and hypertension were associated with increased mortality in patients, as shown in Table 2. Furthermore, the symptoms of cough and fever had a negative association with mortality. Multiple logistic regression adjusted for confounding factors demonstrated that the variables reported in Table 3 , including age, smoking, decreased consciousness at the time of admission, history of cardiovascular disease, and dyspnea, were associated with mortality. Also, the symptoms of cough and fever had a negative association with mortality.

Table 2. Factors Associated with Mortality in Patients with Coronavirus Disease 2019 Using Simple Logistic Regression ${ }^{\mathrm{a}}$

\begin{tabular}{lccc}
\hline Characteristics & OR & 95\% CI & P Value \\
\hline Age & 1.39 & $1.02-1.06$ & $0.001^{\mathrm{b}}$ \\
\hline Sex & 1.51 & $0.71-3.22$ & 0.281 \\
\hline Current smoking & 11.44 & $1.01-129.53$ & $0.049^{\mathrm{b}}$ \\
\hline Diabetes & 1.58 & $0.32-7.94$ & 0.574 \\
\hline Hypertension & 5.82 & $1.13-30.04$ & $0.035^{\mathrm{b}}$ \\
\hline Cardiovascular disease & 5.07 & $1.46-17.58$ & $0.010^{\mathrm{b}}$ \\
\hline At least one underlying disease & 3.31 & $1.54-7.09$ & $0.002^{\mathrm{b}}$ \\
\hline Fever & 0.278 & $0.10-0.74$ & $0.011^{\mathrm{b}}$ \\
\hline Cough & 0.383 & $0.17-0.88$ & $0.023^{\mathrm{b}}$ \\
\hline Dyspnea & 1.63 & $0.79-3.36$ & 0.185 \\
\hline Loss of consciousness & 24.23 & $2.62-223.39$ & $0.005^{\mathrm{b}}$ \\
\hline More than one sign or symptom & 0.467 & $0.23-0.97$ & $0.041^{\mathrm{b}}$ \\
\hline Hospital stay & 0.818 & $0.66-1.01$ & 0.062 \\
\hline Abbreviation $\mathrm{Cl}$ ( & & & \\
\hline
\end{tabular}

Abbreviation: $\mathrm{CI}$, confidence interval.

${ }^{a}$ Qualitative variables are reported as numbers (percentages) and quantitative variables as mean $\pm S D$ or median (IQR).

${ }^{\mathrm{b}}$ Significant at the 0.05 level.

According to the results, all patients had pneumonia with abnormal findings on chest CT scans, so that CT scan evidence of COVID-19 was detected in 100\% (245/245) of patients. The RT-PCR test results were positive in 87 (35.50\%) patients (38.7\% in males and 30.0\% in females $(\mathrm{P}=0.108)$. The results of the chest CT scan stratified by positive or negative RT-PCR tests are shown in Table 4. The sensitivity of chest CT scan and RT-PCR tests in detecting COVID-19 was 100\% (245/245) and 35.5\% (87/245), respectively. In general, our findings showed that the sensitivity of the chest CT scan was much greater than that of the RT-PCR test. 
Table 3. Factors Associated with Mortality in Patients with Coronavirus Disease 2019 Using Multiple Logistic Regression Model

\begin{tabular}{lccc}
\hline Characteristics & OR & 95\% CI & P Value \\
\hline Age & 1.04 & $1.02-1.06$ & $0.002^{\mathrm{a}}$ \\
\hline Smoking & 13.65 & $1.14-17.57$ & $0.039^{\mathrm{a}}$ \\
\hline Cardiovascular disease & 4.48 & $1.14-17.61$ & $0.032^{\mathrm{a}}$ \\
Fever & 0.388 & $0.14-1.11$ & 0.078 \\
\hline Cough & 0.336 & $0.13-0.86$ & $0.023^{\mathrm{a}}$ \\
\hline Dyspnea & 2.43 & $1.03-5.70$ & $0.041^{\mathrm{a}}$ \\
\hline Loss of consciousness & 12.20 & $1.20-23.50$ & $0.034^{\mathrm{a}}$ \\
\hline
\end{tabular}

Abbreviation: $\mathrm{CI}$, confidence interval.

${ }^{\mathrm{a}}$ Significant at the 0.05 level.

The typical findings of radiographic features in chest CT imaging of two COVID-19 patients at the ICU showed bilateral ground-glass opacities in the peripheral zones of LUL and RUL with pleural sparing distribution, as well as small round-shaped ground-glass opacities with peribronchovascular distribution in RUL, as depicted in Figure 3, while later chest CT images showed ground-glass opacities in the peripheral zone of LUL with pleural sparing distribution.

\section{Discussion}

In January 2020, a catastrophic outbreak of COVID-19 occurred in Wuhan, China. The causative agent was a coronavirus (SARS-CoV-2) that is mainly transmitted through respiratory droplets and direct contact. Due to the deadly pulmonary complications of the disease, non-specificity of the symptoms, and lack of effective treatments, the early diagnosis and treatment of the disease and the isolation of patients are very important for disease control.

In the present study, 63\% of the patients were men, which is similar to other studies reporting that the highest percentage of patients belonged to men (13,16-19). One of the causes could be female hormones, which can play a protective role against COVID-19 (20).

In the current study, the mean age of the patients was 54.68 years compared to a study by Chen et al. (17) that reported the age of 55.5 years, Pan et al. (21) that reported 40 years, and Fang et al. (13) that reported 45 years. The rate of fever as the presenting symptom of the patients was $31.8 \%$ in our study, while in other studies, it was reported as $61 \%$ to $98 \%$ (16-19). Besides, $55.9 \%$ of the patients had dyspnea, which was reported to be 55\% (16) and 31\% (19) in other studies. In $38 \%$ of the patients, the cough symptom was observed, which was estimated at 79\% (22), 76\% (16), 82\% (17), $59 \%$ (19), and 48\% (18) in other studies. The most common symptoms in this study were fever and cough. This finding has also been reported in other studies. In addition, a small percentage of patients reported symptoms such as diarrhea, which was observed in other studies, as well (16, 17). According to the results of recent studies, diarrhea has been a common symptom of SARS-CoV and MERS-CoV infection (23).

In this study, $20 \%$ of the patients were admitted to the ICU. This rate was $31 \%$ in a study by Huang et al. (16). However, in a study conducted by Wang et al. (19), this rate was $26 \%$. The reason for the lower percentage of patients admitted to the ICU in our study could be the inadequate number of beds in the ICU. There was no significant difference between the age of patients admitted to the ICU and nonICU wards, which is consistent with a study by g et al. (16). However, Wang et al. (19) reported a significant difference between the age of patients admitted to the ICU and nonICU wards.

In the present study, the mortality rate was $15.5 \%$, while other previous studies showed the mortality rates of 11 to $28 \%$ in all patients with COVID-19 $(16,17,24)$. Given that about $81 \%$ of the patients with COVID-19 have mild diseases and never require hospitalization, the mortality rate of 15.5 $\%$ in hospitalized patients would be reasonable.

This study identified several factors associated with mortality in patients who were hospitalized with COVID19. In particular, age, smoking, the presenting symptom of dyspnea, decreased consciousness at the time of admission, and history of cardiovascular disease were associated with higher odds of in-hospital mortality. In some studies, older age was reported as a factor associated with mortality in SARS and MERS (25). The current study confirmed that old age was associated with mortality in patients with COVID-19, which is consistent with the findings of previous studies $(22,24,26)$. In the univariate analysis, underlying diseases such as cardiovascular disease and hypertension were associated with mortality, as was reported similarly by Jordan (24).

W observed that the prevalence of smoking and underlying diseases in patients with COVID-19 was lower than estimated in the general population (27); this could be explained by defects or inaccuracy in the recorded data or the fact that high-risk people were less likely to be exposed to the virus due to taking proper preventive care.

The gold standard for the diagnosis of COVID-19 is RTPCR, which has significant false-negative results and is time-consuming. Therefore, chest CT scans could be helpful in the early detection of lung involvement and diagnosis of the disease. In this study, the sensitivity of chest CT scan and RT-PCR was $100 \%$ and 35.5\%, respectively. The low sensitivity of the RT-PCR test could be due to the defect in proper oropharyngeal sampling or the low sensitivity of 


\begin{tabular}{|c|c|c|}
\hline \multirow{2}{*}{ Radiographic Features in CT Scan of COVID-19 Patients } & \multicolumn{2}{|c|}{ RT-PCR, \% } \\
\hline & Positive, $(\mathbf{N}=87)$ & Negative, $(N=158)$ \\
\hline 1. Ground-glass opacities (bilateral, sub-pleural, peripheral) & 89.7 & 90.8 \\
\hline $\begin{array}{l}\text { 2. Ground-glass opacities and interlobular septal thickening, crazy-paving } \\
\text { patterns }\end{array}$ & 18.9 & 23.1 \\
\hline 3. Air space consolidation & 58.3 & 43.1 \\
\hline 4. Bronchovascular thickening in the lesion & 17.3 & 8.1 \\
\hline 5. Traction bronchiectasis & 3.1 & 1.9 \\
\hline 6. Normal CT scan & 0 & 0 \\
\hline 7. Pleural effusion & 1.5 & 0.7 \\
\hline 8. Tree-in-bud pattern & 1 person & - \\
\hline 9. Pneumothorax & 0 & 0 \\
\hline 10. Cavitation & 0 & 0 \\
\hline 11. Mediastinal lymphadenopathy & 0 & 0 \\
\hline
\end{tabular}
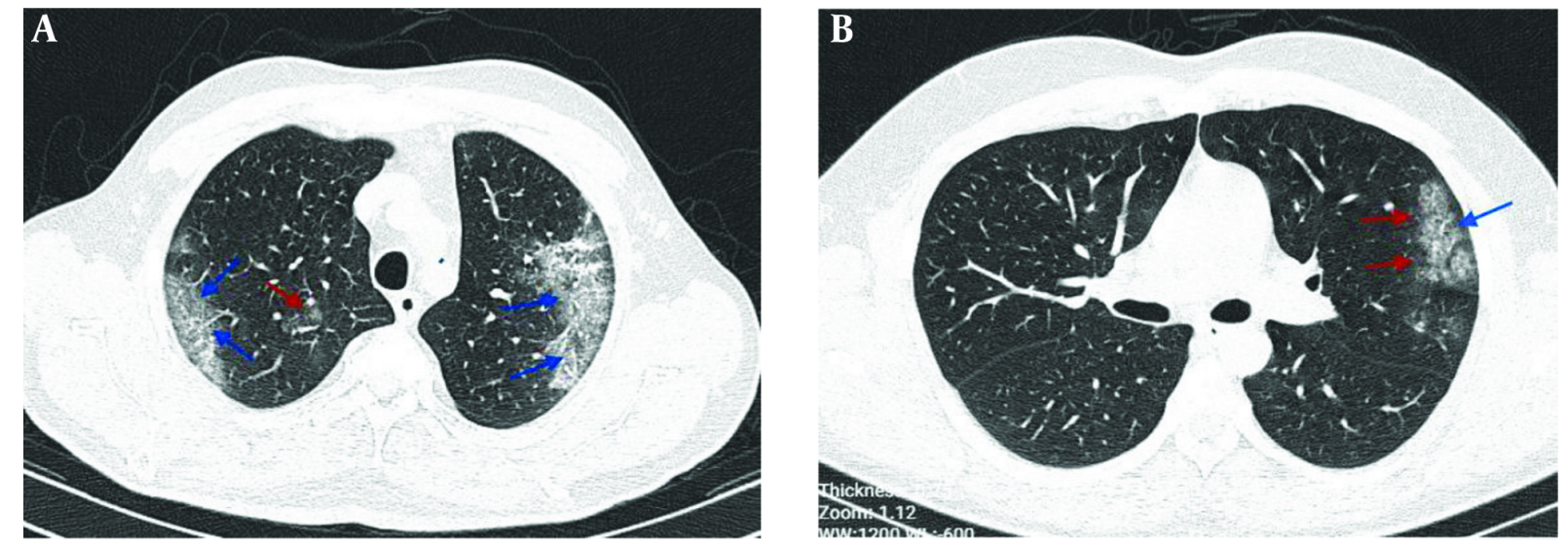

Figure 3. Chest CT images; A, an axial CT scan image without contrast in a 53-year-old male patient with cough, fever, and dyspnea, showing bilateral ground-glass opacities in the peripheral zones of LUL and RUL with pleural sparing distribution (blue arrows) and small round-shape ground-glass opacities with peribronchovascular distribution in RUL (red arrow); B, an axial CT scan image without contrast in a 29-year-old male patient with fever and dyspnea showing ground-glass opacities in the peripheral zone of LUL (red arrows) with pleural sparing distribution (arrow).

the test kit.

The most common finding in chest CT scans of patients with positive and negative RT-PCR tests in our study was ground-glass opacities. Pulmonary ground-glass involvement was bilateral in all of the patients, with the peripheral areas more affected. The pathological explanation of this finding could be inflammation and infiltration of inflammatory cells, exudative fluid secretion, or bleeding into the interstitial tissue of the lungs (28). The absence of pneumothorax, mediastinal adenopathy, cavitation, and rarity of pleural effusion, pulmonary nodularity, and bronchiectasis in patients with coronary artery disease indicate that the appearance of any of these findings is detrimental to the diagnosis, and another differential di- agnosis should be considered.

Influenza A (H1N1) and adenovirus pneumonia could manifest radiologically as COVID-19 and should be differentiated from COVID-19. Chest CT scan findings may be helpful to differentiate these important diseases. In adenoviral pneumonia, the involvement of the central lung area, along with pleural effusion, pneumothorax, and widening of pulmonary hilum, is prominent (29), while $\mathrm{H} 1 \mathrm{~N} 1$ pneumonia causes the appearance of ground-glass opacities and consolidations, with a peribronchovascular predominance (30).

However, this study had two limitations. First, the data were collected for clinical purposes, not for systematic use; thus, some cases had incomplete documentation, which 
may be inappropriate for research purposes. The second limitation was the fact that asymptomatic or mild patients were treated at home; therefore, they were not included in the study, and only symptomatic people with more severe disease were studied, so the results might have been biased.

\section{Footnotes}

Authors' Contribution: SV, ZI, and ZF developed the idea, designed the study, had full access to all data in the study, and take responsibility for the integrity of the data, and the accuracy of data analysis. ZF, ZI, SJ, HD, SV, MS, AJ, BS, MS, SVJ, FM, ZM, and RM contributed to the writing of this report. ZI and ZF contributed to the critical revision of the report. ZF contributed to statistical analysis. All authors contributed to data acquisition, data analysis, or data interpretation, and reviewed and approved the final version.

Conflict of Interests: All authors declare that they have no conflict of interest.

Funding/Support: This work was funded by the Kermanshah University of Medical Sciences (KUMS).

\section{References}

1. Cascella M, Rajnik M, Cuomo A, Dulebohn SC, Di Napoli R. Features, Evaluation, and Treatment of Coronavirus. StatPearls. Treasure Island (FL); 2020.

2. Han Q, Lin Q, Jin S, You L. Coronavirus 2019-nCoV: A brief perspective from the front line. I Infect. 2020;80(4):373-7. doi: 10.1016/j.jinf.2020.02.010. [PubMed: 32109444]. [PubMed Central: PMC7102581].

3. Sohrabi C, Alsafi Z, O'Neill N, Khan M, Kerwan A, Al-Jabir A, et al. World Health Organization declares global emergency: A review of the 2019 novel coronavirus (COVID-19). Int J Surg. 2020;76:71-6. doi: 10.1016/j.ijsu.2020.02.034. [PubMed: 32112977]. [PubMed Central: PMC7105032].

4. WHO. Novel coronavirus(2019-nCoV). 2020. Available from: https://www.who.int/docs/default-source/coronaviruse/situationreports/20200130-sitrep-10-ncov.pdf?sfvrsn=d0b2e480_2.

5. Guo W, Li M, Dong Y, Zhou H, Zhang Z, Tian C, et al. Diabetes is a risk factor for the progression and prognosis of COVID-19. Diabetes Metab Res Rev. 2020. e3319. doi:10.1002/dmrr.3319. [PubMed: 32233013]. [PubMed Central: PMC7228407].

6. Shi S, Qin M, Shen B, Cai Y, Liu T, Yang F, et al. Association of cardiac injury with mortality in hospitalized patients with COVID-19 in Wuhan, China. JAMA Cardiol. 2020;5(7):802-10. doi: 10.1001/jamacardio.2020.0950. [PubMed: 32211816]. [PubMed Central: PMC7097841].

7. Lai J, Ma S, Wang Y, Cai Z, Hu J, Wei N, et al. Factors associated with mental health outcomes among health care workers exposed to coronavirus disease 2019. JAMA Netw Open. 2020;3(3). e203976. doi: 10.1001/jamanetworkopen.2020.3976. [PubMed: 32202646]. [PubMed Central: PMC7090843]

8. Xiao X, Liu Q, Zhu X, Li Y, Li X. Risk factors associated with hospitalacquired infections for COVID-19 patients in ICU. SSRN Electron J. 2020. doi: $10.2139 /$ ssrn.3564427.

9. WHO. Laboratory testing for coronavirus disease 2019 (COVID-19) in suspected human cases: interim guidance, March 2 2020. 2020. Available from: https://apps.who.int/iris/handle/10665/331329.
10. Corman V, Bleicker T, Brünink S, Drosten C, Zambon M. Diagnostic detection of 2019-nCoV by real-time RT-PCR. World Health Organization. 2020;17.

11. Rahimzadeh G, Ekrami Noghabi M, Kadkhodaei Elyaderani F, Navaeifar MR, Enayati AA, Manafi Anari A, et al. Covid-19 infection in Iranian children: A case series of 9 patients. JPediatrRev. 2020;8(2):139-44. doi 10.32598/jpr.8.2.139.

12. Ai T, Yang Z, Hou H, Zhan C, Chen C, Lv W, et al. Correlation of chest CT and RT-PCR testing for coronavirus disease 2019 (COVID19) in China: A report of 1014 cases. Radiology. 2020;296(2):E32-40. doi: 10.1148/radiol.2020200642. [PubMed: 32101510]. [PubMed Central: PMC7233399].

13. Fang Y, Zhang H, Xie J, Lin M, Ying L, Pang P, et al. Sensitivity of chest CT for COVID-19: Comparison to RT-PCR. Radiology. 2020;296(2):E1157. doi: 10.1148/radiol.2020200432. [PubMed: 32073353]. [PubMed Central: PMC7233365].

14. Ye Z, Zhang Y, Wang Y, Huang Z, Song B. Chest CT manifestations of new coronavirus disease 2019 (COVID-19): a pictorial review. Eur Radiol. 2020;30(8):4381-9. doi: 10.1007/s00330-020-06801-0. [PubMed: 32193638]. [PubMed Central: PMC7088323].

15. Shi H, Han X, Jiang N, Cao Y, Alwalid O, Gu J, et al. Radiological findings from 81 patients with COVID-19 pneumonia in Wuhan, China: a descriptive study. Lancet Infect Dis. 2020;20(4):425-34. doi: 10.1016/s14733099(20)30086-4.

16. Huang C, Wang Y, Li X, Ren L, Zhao J, Hu Y, et al. Clinical features of patients infected with 2019 novel coronavirus in Wuhan, China. Lancet. 2020;395(10223):497-506. doi: 10.1016/S0140-6736(20)30183-5. [PubMed: 31986264]. [PubMed Central: PMC7159299].

17. Chen N, Zhou M, Dong X, Qu J, Gong F, Han Y, et al. Epidemiological and clinical characteristics of 99 cases of 2019 novel coronavirus pneumonia in Wuhan, China: a descriptive study. Lancet. 2020;395(10223):507-13. doi: 10.1016/S0140-6736(20)30211-7. [PubMed: 32007143]. [PubMed Central: PMC7135076].

18. Bernheim A, Mei X, Huang M, Yang Y, Fayad ZA, Zhang N, et al Chest CT findings in coronavirus disease-19 (COVID-19): Relationship to duration of infection. Radiology. 2020;295(3):200463. doi 10.1148/radiol.2020200463. [PubMed: 32077789]. [PubMed Central PMC7233369].

19. Wang D, Hu B, Hu C, Zhu F, Liu X, Zhang J, et al. Clinical characteristics of 138 hospitalized patients with 2019 novel coronavirusinfected pneumonia in Wuhan, China. JAMA. 2020;323(11):1061-9. doi: 10.1001/jama.2020.1585. [PubMed: 32031570]. [PubMed Central: PMC7042881].

20. Moxley G, Posthuma D, Carlson P, Estrada E, Han J, Benson LL, et al. Sexual dimorphism in innate immunity. Arthritis Rheumatol. 2002;46(1):250-8. doi: 10.1002/1529-0131(200201)46:1<250::aidart10064>3.0.co;2-t.

21. Pan F, Ye T, Sun P, Gui S, Liang B, Li L, et al. Time course of lung changes at chest CT during recovery from coronavirus disease 2019 (COVID19). Radiology. 2020;295(3):715-21. doi: 10.1148/radiol.2020200370 [PubMed: 32053470]. [PubMed Central: PMC7233367].

22. Zhou F, Yu T, Du R, Fan G, Liu Y, Liu Z, et al. Clinical course and risk factors for mortality of adult inpatients with COVID-19 in Wuhan, China: a retrospective cohort study. Lancet. 2020;395(10229):1054-62. doi: 10.1016/s0140-6736(20)30566-3.

23. Assiri A,Al-Tawfiq JA,Al-Rabeeah AA,Al-Rabiah FA, Al-Hajjar S, Al-Barrak A, et al. Epidemiological, demographic, and clinical characteristics of 47 cases of Middle East respiratory syndrome coronavirus disease from Saudi Arabia: a descriptive study. Lancet Infect Dis. 2013;13(9):75261. doi: 10.1016/S1473-3099(13)70204-4. [PubMed: 23891402]. [PubMed Central: PMC7185445].

24. Jordan RE, Adab P, Cheng KK. Covid-19: risk factors for severe disease and death. BMJ. 2020;368:m1198. doi: 10.1136/bmj.m1198. [PubMed: 32217618]. 
25. Peeri NC, Shrestha N, Rahman MS, Zaki R, Tan Z, Bibi S, et al. The SARS, MERS and novel coronavirus (COVID-19) epidemics, the newest and biggest global health threats: what lessons have we learned? Int J Epidemiol. 2020;49(3):717-26. doi: 10.1093/ije/dyaa033. [PubMed: 32086938]. [PubMed Central: PMC7197734].

26. Sarkar J, Chakrabarti P. A machine learning model reveals older age and delayed hospitalization as predictors of mortality in patients with COVID-19. medRxiv. 2020. doi:10.1101/2020.03.25.20043331.

27. World Life Expectancy. Iran: Life expectancy. 2020. Available from: https://www.worldlifeexpectancy.com/iran-life-expectancy.

28. Gotway MB, Reddy GP, Webb WR, Elicker BM, Leung JW. Highresolution CT of the lung: patterns of disease and differen- tial diagnoses. Radiol Clin North Am. 2005;43(3):513-42. viii. doi: 10.1016/j.rcl.2005.01.010. [PubMed: 15847814].

29. Tan D, Zhu H, Fu Y, Tong F, Yao D, Walline J, et al. Severe communityacquired pneumonia caused by human adenovirus in immunocompetent adults: A multicenter case series. PLoS One. 2016;11(3). e0151199. doi: 10.1371/journal.pone.0151199. [PubMed: 26967644]. [PubMed Central: PMC4788423].

30. Li P, Zhang JF, Xia XD, Su DJ, Liu BL, Zhao DL, et al. Serial evaluation of high-resolution CT findings in patients with pneumonia in novel swine-origin influenza A (H1N1) virus infection. Br J Radiol. 2012;85(1014):729-35. doi: 10.1259/bjr/85580974. [PubMed: 22167502]. [PubMed Central: PMC3474108]. 